\title{
ARM Cortex Based Industrial Data Acquisition System with Smart Wireless Sensor Interface based on Internet of Things using WiFi Network
}

\author{
Mrs Hemalatha $\mathbf{B}^{1}$, Prabhakar ${ }^{2}$ \\ VLSI design and embedded system Engineering Bellary Institute of Technology and Management, Bellary, India ${ }^{1}$ \\ Electronics and Communication Engineering, Bellary Institute of Technology and Management, Bellary, India ${ }^{2}$
}

\begin{abstract}
Internet plays a vital role in our day to day life. without internet we cannot proceed our lives normally. In Internet also there are types one is wireless and other is wired. In that we prefer almost all wireless Internet since we can access it from anywhere. Here the purpose is to monitor and manage the environmental changes occuring using various sensors like humidity sensor, temperature sensor, smoke sensor and light dependent resistor. Here also using automatic ON OFF cooling fan if there is an any increase in temperature, smoke. This can be performed using ARMCortex M3 because of low power consumption. Here using wifi network and is connected to laptop for uploading database using bluetooth module with time and date. By this we can see the uploaded database at any time. this is used in industries, power plants, mining and so on.
\end{abstract}

\section{INTRODUCTION}

Now a days there is an rapid growth in environmental change. To manage and monitor the environmental changes has become a complex tasks for both scientists and engineers and so on. If we considering the application range of various techniques, the cost which is involved in the data transmission, security, reliability and other various parameters, Bluetooth and wireless sensor networks communication are the most absolute methods in the short range wireless communication and data transmission.

Environmental science information's which involves specific environmental science problems related to the applications of engineering techniques, management information systems and environmental information systems, which were designed to collect, process, and exchange the data and information.

Data acquisition is used to collect data and measure some physical quantities such as smoke, temperature, humidity. All these sensors output values are in analog. To convert these output analog values we are using analog to digital conversion unit. Embedded system or unit consists of various sensors such as humidity, smoke, temperature, light dependent resistor. and also consists of ARM-Cortex M3 controller which is the latest controller and uses less power.

The cooling fan manages or controls increase in temperature and smoke. this is one advantages in industries because of automatic controlling cooling fan. This embedded unit is connected via bluetooth module to the laptop which is acting as local server. For uploading the smoke, temperature, humidity and LDR values, wifi network is connected via android mobile phone and uploads the data values in database with date and time. Because of the busy schedules, the owner can go through uploaded data at any time.

\section{BLOCK DIAGRAM}

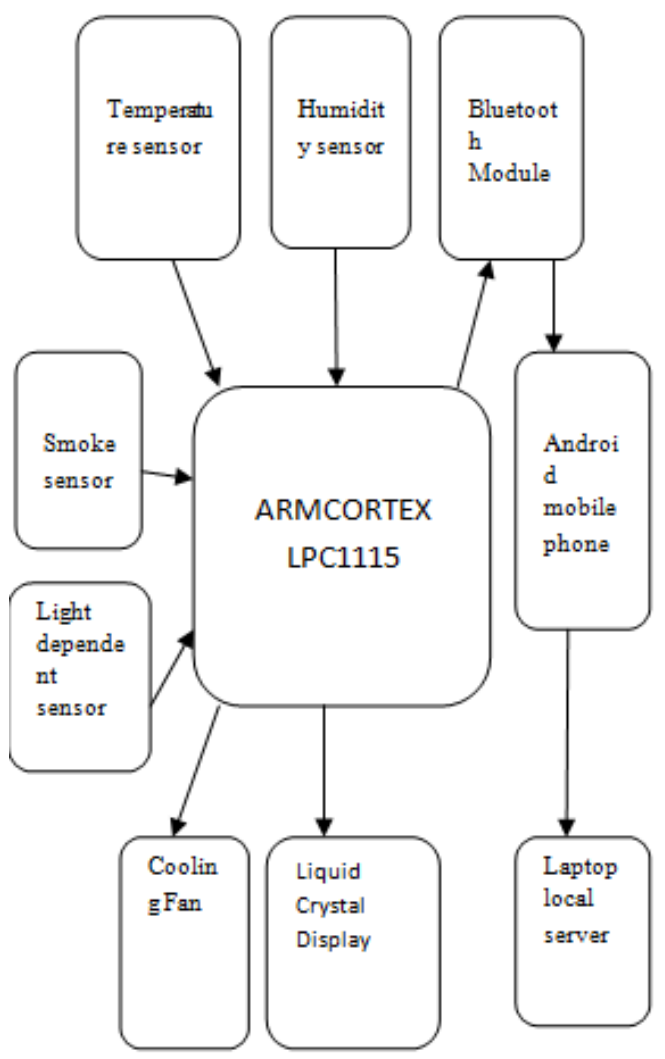

III. LITERATURE SURVEY

In earlier days if we want access anything from internet we should go the internet centers. But, now a days we have many sources to access like using modems or using android mobile phones. From last two to three years we have wifi hotspot option so by that we can connect to any other mobile phone or laptops. This is the major advantage for users. In thing paper we are also using ARM Cortex M3 i.e, LPC1115 which is the latest version of ARM 
Cortex controller which consumes less power that is $3.3 \mathrm{v}$ smoke, humidity and LDR values with time and date and and frequency is $12 \mathrm{MHz}$. In this paper, latest version maintains the data on the local server. Java coding using controller and internet technology are used which makes Eclipse, along with Android 4.0 SDK is used to create the easier to access. Industrial information engineering is front-end of the server. used to integrate and coordinate various information technologies components and also it facilitates the decision making to link the territorial knowledge with the environmental objectives and also handle some problems related to environmental conditions which are more cost effective. In this work multiple sensors and recent technologies are used to make easier for various industries purpose, home appliances, power plants and so on.

\section{Components List}

- ARM Cortex M3 LPC115.

- Temperature Sensor.

- Humidity Sensor.

- Smoke Sensor.

- Light Dependent Resistor(LDR).

- Bluetooth module(HC06)

- Android mobile phone.

- LCD.

- Cooling fan.

- Laptop.

\section{Software's Used}

- Coocox IDE

- Coocox Flash programmer.

- Eclipse.

- $\quad$ Android 4.0.

- MySQL.

\section{DESCRIPTION}

Embedded units consists ARM Cortex M3 and various sensors like temperature, smoke, humidity and light dependent resistor. It also consists of cooling fan which is automatic ON OFF to control the increase in temperature and smoke. Android mobile phone is connected via bluetooth module to embedded unit. A same network is provided to both mobile mobile phone and laptop using wifi hotspot. The temperature sensor is used to check for any change in temperature. The humidity sensor is used to check for any change in humidity within the warehouse or cold storage facility, whereas smoke sensor is used to detect gases coming from decaying food or to check any gas leakage is there. LDR is a variable resistor whose value decreases with increase in incident light intensity. All these sensors output values are in analog. To convert these output analog values an analog to digital conversion unit is used. Automatic ON OFF cooling fan is ON when there is increase in smoke and temperature or else it will in off condition.

The microcontroller processes the incoming voltages from the sensors depending on a program embedded within that. The output of the microcontroller is given to Android mobile phone via Bluetooth. From the Android mobile phone the data is then uploaded to the local server laptop via Wi-Fi network. A database is created using MySQL software, which contains details about temperature,

\section{APPLICATION}

- It is used in industries such as food processing management.

- It is used in nuclear power plant.

- It is used in small scale offices and in homes.

- It is used in building management and in health care management.

\section{CONCLUSION}

In this paper, we can conclude that it improves the efficiency of environmental monitoring and management tasks based on internet of things. It benefits not only data acquisition but also benefits in web service applications such as cloud computing and environmental sciences. This is also valuable for transformation perception, management, processing, and sharing of multisource information in environmental sciences. This unit is used in anywhere as mentioned in applications.

\section{REFERENCES}

[1]. E. Welbourne, L. Battl "Building the Internet of things using RFID: The RFID ecosystem experience"

[2]. N. Niu, L. Xu, and Z. Bi, "Enterprise information systems architecture analysis and evaluation.

[3]. M. Haklay, From Environmental Information Systems to Environmental Informatics

[4]. L. Xu, Z. Li, S. Li, and F. Tang, "A decision support system for product design in concurrent engineering"

[5]. N. M. Avouris and B. Page, in Environmental Informatics: Methodology and Applications of Environmental Information Processing. 